\title{
Counterfactual thinking about controllable events
}

\author{
RACHEL MCCLOY and RUTH M. J. BYRNE \\ University of Dublin, Trinity College, Ireland
}

\begin{abstract}
When people think about what might have been, they mentally undo controllable rather than uncontrollable events. We report the results of two experiments in which we examined this controllability effect in counterfactual thinking. The experiments show that the mutability of controllable events is influenced by the perceived appropriateness or inappropriateness of the events. The first experiment shows that people change inappropriate controllable actions more than appropriate controllable ones. The second experiment shows that people mutate inappropriate controllable events whether the outcome is exceptional or normal with respect to intrapersonal habitual norms, and whether the outcome is positive or negative. We discuss the implications for alternative theories of counterfactual thinking.
\end{abstract}

When people think back over the events of their lives and the lives of others, they often think "if only ...," and they construct counterfactual alternatives about what might have been. Such counterfactual thinking is pervasive in everyday life, and it has been examined by philosophers and psychologists interested in counterfactual reasoning (e.g., Byrne \& Tasso, 1999; Lewis, 1973; Stalnaker, 1968) and in the generation of counterfactual scenarios (e.g., Kahneman \& Tversky, 1982; Roese, 1997; Roese \& Olson, 1995). The set of alternatives to any situation is infinite. Some of the alternatives are based on small changes to reality (e.g., "if only I had arrived one minute earlier ..."), and some are based on greater changes (e.g., "if only I had been born 100 years earlier ..."). In everyday counterfactual thinking, people may have neither the time nor the cognitive capacity to consider every possible alternative. Instead, the alternatives that come to mind may be constrained by the aspects of reality that are most readily mutated, such as the causal relations between events (e.g., Wells, Taylor, \& Turtle, 1987), their temporal relations (Byrne, Segura, Culhane, Tasso, \& Berrocal, 2000; Miller \& Gunasegaram, 1990), their normality or exceptionality (Kahneman \& Tversky, 1982), and their controllability (Girotto, Legrenzi, \& Rizzo, 1991). In this paper, we aim to examine further the mutability of controllable and uncontrollable events in the generation of counterfactual scenarios.

\footnotetext{
We thank Sean Commins, Colin Gemmell, Vittorio Girotto, Simon Handley, Mark Keane, Mick O'Connell, Alice McEleney, Susana Segura, and Clare Walsh for their helpful comments. Some of the results were reported at the Fifth International Colloquium on Cognitive Science, San Sebastian, Spain in 1997 and at the Twenty-Eighth Annual Conference of the Psychological Society of Ireland in 1997. The first author is supported by a postgraduate studentship from the Department of Education for Northern Ireland. Correspondence should be addressed to R. McCloy, Psychology Department, University of Dublin, Trinity College, Dublin 2, Ireland (e-mail: mccloyr@tcd.ie).
}

Controllable events are more mutable than uncontrollable events (Girotto et al., 1991). People tend to mentally undo actions that are controllable, such as actions that result from an intentional decision. Girotto et al. gave their participants a scenario about a Mr. Bianchi, who was delayed on his way home from work by four events, with the outcome that he arrived home too late to save his wife, who had a fatal heart attack. Three of the delaying events were outside his control, and they included external events such as a fallen tree blocking the road, as well as events constrained by circumstances such as Mr. Bianchi's having an asthma attack or returning to the office for a spare pair of glasses after breaking his current pair. The controllable event was an intentional decision by Mr. Bianchi to have a beer in a bar. Participants asked to complete Mr. Bianchi's "if only ..." thoughts tended to undo the controllable event more than the uncontrollable events, saying, for example, "if only I hadn't stopped for a drink then my wife would be alive." They undid the controllable event more than the uncontrollable events, whether the uncontrollable events were external events or actions of the protagonist constrained by circumstances; they undid the controllable event whether it was framed as normal or exceptional; and they undid it regardless of the order in which it occurred in the sequence of events. Girotto et al.'s findings suggest that there is a controllability effect in counterfactual thinking: Controllable events are more mutable than uncontrollable events (see also Mandel \& Lehman, 1996).

Uncontrollable events also differ in their mutability. Girotto et al. (1991, Experiment 3) found differences in the frequency with which uncontrollable events were undone: People mentally undid Mr. Bianchi's return for a spare pair of glasses more often than his stop for an asthma attack. Do controllable events also differ in their mutability? Our aim in this paper is to address this question. The only controllable event that Girotto et al. pitted against uncontrollable events was Mr. Bianchi's decision to drink a 
beer in a bar. Are all controllable events equally mutable? We suspect that some controllable events may be more psychologically mutable than others. Consider Mr. Bianchi's tendency to think that he could have saved his wife if only he had not called into the bar for a drink. Suppose that he had been delayed instead because he decided to call in to his aging parents to check whether or not they were well - an equally controllable event. Would there be an equally strong tendency to think he could have saved his wife if only he had not called in to check on his parents? These two sorts of controllable events may differ on many dimensions, but we suggest that one important difference is in what we will call their "appropriateness." An event may be appropriate or inappropriate within a particular context: Drinking in a bar may be considered to be appropriate in the context of celebrating with friends; it may be considered to be inappropriate in the context of arriving home too late to save a dying spouse. We suggest that inappropriate controllable events may be more psychologically mutable than appropriate controllable events: A protagonist can more easily wish that he had not done something inappropriate than he can wish that he had not done something appropriate.

Why might inappropriate events be more psychologically mutable than appropriate events? One suggestion is that inappropriate controllable events may be more mutable than appropriate controllable events because inappropriate events are exceptional and appropriate events are normal. The definitions of normality and exceptionality put forward by Kahneman and Miller (1986) could potentially include those of appropriateness and inappropriateness. Appropriate events may be said to be normal from the perspective of societal norms for behavior, whereas inappropriate events may instead be said to be exceptional. Kahneman and Miller's conception of normality is quite general, and other researchers who have examined the exceptionality effect in counterfactual thinking have used more limited definitions of norms and exceptions (e.g., Gavanski \& Wells, 1989; Girotto et al., 1991; Kahneman \& Tversky, 1982). They have operationally defined norms and exceptions intrapersonally as adherence to or deviations from a person's habitual modes of behavior. Interestingly, Girotto et al. showed that, regardless of whether the controllable event in their scenario was described as normal or exceptional for the protagonist, it was still undone significantly more often than any of the uncontrollable events described in the scenario. They did not find a significant difference between the mutability of controllable events when they were described as normal and when they were described as exceptional for the protagonist.

The increased mutability of controllable events over uncontrollable events is not affected by whether they are exceptional or normal with respect to intrapersonal norms. What about interpersonal, societal norms? Seelau, Seelau, Wells, and Windschitl (1995) have argued that counterfactual mutations are influenced by a range of differ- ent kinds of constraints. For example, they suggest that "people's implicit knowledge of the constant effects of the basic laws of science and nature, such as gravity ... constrains the counterfactuals they generate" (p. 60). We suggest that, not only the understanding of natural laws, but also the understanding of social norms and expectations, can act to constrain the counterfactuals that people generate. Potential social constraints in counterfactual thinking may include the appropriateness of actions, their selfishness, impulsivity, and so on. In this paper we will focus on one possible social constraint in counterfactual thinking, whether actions are appropriate or inappropriate - that is, the effect of interpersonal (rather than intrapersonal) normality on the mutability of controllable events. In the first experiment, we examined whether varying the appropriateness of controllable events (i.e., whether they were exceptional or normal with respect to interpersonal norms) would affect their mutability. In the second experiment, we examined whether the effects of such social constraints-that is, interpersonal norms - are distinct from the effects of intrapersonal norms (i.e., whether the behavior is normal or exceptional for the individual in question).

Mr. Bianchi's decision to have a beer in a bar is a controllable event that may be construed as inappropriate. It may be undone more often not merely because it is a controllable event, but because it is an inappropriate controllable event. Support for this suggestion exists in N'gbala and Branscombe's (1995) finding that people more readily undo events for which there are "immoral" reasons. N'gbala and Branscombe varied the reasons why an individual, Joe, was late picking up his child from school. The child got a lift home from a neighbor instead and was seriously injured when the neighbor's car was involved in a collision with a drunk driver. In one version of the scenario, Joe was late because he had stopped to help someone (N'gbala and Branscombe call this decision a "moral reason"; we prefer the more contextually specific terminology of an "appropriate controllable" event). In a second version, Joe was late because he had stopped to talk with friends (in N'gbala and Branscombe's terminology, an "immoral reason"; in ours, an "inappropriate controllable" event). N'gbala and Branscombe found that participants assigned more fault to Joe and mutated his behavior more frequently when the reasons for his behavior were immoral. In the two experiments that we report, we tested further the effect of appropriateness on the mutability of controllable events.

\section{EXPERIMENT 1}

Our aim in the first experiment was to examine the mutability of controllable events that differed in their appropriateness. We expected that an inappropriate controllable event would be more mutable than an uncontrollable event, as Girotto et al. (1991) have demonstrated. However, we also wished to compare different controllable events to see whether an inappropriate controllable event would be 
Table 1

The Percentages of Mutations of the Target Events in Experiment 1

\begin{tabular}{cc}
\hline Event & First Alternatives \\
\hline Controllable & \\
Inappropriate & 22 \\
Neutral & 11 \\
Appropriate & 12 \\
Uncontrollable & 9 \\
Delay & 36 \\
\hline
\end{tabular}

more mutable than either an appropriate controllable event or a neutral controllable event. Finally, we also wished to see whether an appropriate controllable event would be more mutable than an uncontrollable event.

\section{Method}

Materials pretest. We used a pretest to generate one appropriate, one neutral, and one inappropriate controllable event to include in the scenario. We examined the appropriateness of 14 events in the context of a delay: going swimming, buying flowers for a girlfriend, drinking with colleagues, playing squash, buying a newspaper, getting a book from the library, watching a football match, renting a video, visiting parents, buying cigarettes, going to the pub, visiting an art exhibition, having a burger, and helping an elderly neighbor.

In the first pretest task, participants were told that they would be given a number of examples of people arriving late when they were expected at a certain time because each had stopped to do something en route to their appointment. The participants were given 14 sets of three-sentence scenarios of the following sort: Fred was late home from work. He had stopped to visit his parents. His wife was angry. They were asked to tick on a 7-point scale from appropriate to inappropriate the appropriateness of the emotional response, which was always either anger or pleasure, with which the lateness was met. The second pretest task consisted of a short passage describing John, who had a limited amount of time and a range of different things that he wanted to do. The participants were given the list of 14 actions from the previous task (in a different random order), and they were asked to rank them in the order in which they believed John should give them priority.

The 20 undergraduate students ( 15 women and 5 men) from the University of Dublin, Trinity College, who took part in the pretest voluntarily were 20 years old on the average. One participant failed to complete the second task. We judged an inappropriate event to be one for which a negative emotional reaction (anger) was an appropriate response to someone delayed by it, and to which a low priority was assigned. The results of the two measures combined showed that the most inappropriate events in the context of being delayed for an appointment were those of stopping to rent a video and stopping to have a hamburger (average combined rank as 1 st and 2nd), the most appropriate events in the context of being delayed for an appointment were those of stopping to buy flowers for a girlfriend and stopping to visit parents (ranked as joint 13th), and the most neutral event in the context of being delayed for an appointment was that of stopping to buy a newspaper (ranked as 8). ${ }^{1}$

Materials and Design. We constructed a scenario based on the one used by Girotto et al. (1991). The four target events were presented in a temporal, noncausal sequence, and they were all framed as exceptional for the protagonist. One event, a traffic jam, was uncontrollable; another, stopping to visit parents, was appropriate and controllable; a third, stopping for a hamburger, was inappropriate and controllable; and the fourth, buying a newspaper, was neutral and controllable. The scenario and the question were as follows:

Mr. Ryan worked in an office several miles from where he and his wife lived. Usually he went straight home from work, however, on the day in question he was delayed for a number of reasons. On leaving the office Mr. Ryan first decided to call and visit his parents who lived nearby. $\mathrm{He}$ then stopped and bought a newspaper. After this he hit rush hour and was delayed in a traffic jam. Following this he pulled into a drivethrough restaurant for a burger. When he finally arrived home Mr. Ryan found his wife on the floor. She had had a heart attack and was dying. He tried to help but his efforts came too late. As commonly happens in such tragic situations Mr. Ryan often thought and often said "if only. .." in the days that followed. How did he complete this thought?

We constructed four versions of the scenario. They differed only in the order of presentation of the four target events: Each event appeared in each position equally often in the scenario, in order to control for order effects. We gave each participant one of the four scenarios at random. The design of the experiment was a fully withinsubjects one, and the independent variable was the nature of the target events: inappropriate controllable, appropriate controllable, neutral controllable, and uncontrollable. The dependent variable was the frequency with which participants mentioned each of the four target events in their first sentence completion.

Participants and Procedure. Seventy-six undergraduates from the University of Dublin, Trinity College (59 women and 17 men) took part in the experiment voluntarily. Their average age was 21 years. They were given a three-page booklet that contained a cover page with instructions, the scenario, and a final page containing a short debriefing paragraph. The instructions asked the participants to read the passage carefully and to provide answers to the question that followed as the answers occurred to them. They were also instructed not to change their answers after they had written them and not to read the explanation until completely finished.

\section{Results and Discussion}

Most participants provided two or three sentence completions of Mr. Ryan's “if only" thoughts. Ninety percent of the first completions mentioned either one of the four target events (54\%) or the overall delay (e.g., "if only I hadn't been delayed"; $36 \%$ ). As Table 1 shows, the results for the first completions provided by each participant indicate that participants undid the inappropriate controllable event more often than the uncontrollable event ( $22 \%$ vs. $9 \%$; binomial, $n=24, z=2.00, p<.05$ ). The result replicates Girotto et al.'s (1991) finding. However, the results also show that participants tended to undo the inappropriate controllable event more often than the appropriate controllable event ( $22 \%$ vs. $12 \%$; binomial, $n=$ $26, z=1.96, p<.05$ ), and they also undid the inappropriate controllable event more often than the neutral controllable event ( $22 \%$ vs. $11 \%$; binomial, $n=25, z=1.8$, $p<.05)$. These results show that there are differences in the mutability of controllable events depending on their appropriateness, as we predicted, and they are consistent with N'gbala and Branscombe's (1995) findings on the influence of morality.

The results also show that participants undo the appropriate controllable event no more often than the uncontrollable event ( $12 \%$ vs. $9 \%$; binomial, $n=16, z=0.5$, $p<.30$ ), they undo the neutral controllable event no more often than the uncontrollable event ( $11 \%$ vs. $9 \%$; binomial, $n=15, z=0.3, p<.40$ ), and they undo the appropriate controllable event as often as the neutral controllable event ( $11 \%$ vs. $12 \%$; binomial, $n=17, z=0.2, p<$ $.40)$. These results show that the event that participants most readily undo is the inappropriate one. The results suggest that the controllability effect may be mediated by 
the appropriateness of events: Participants mentally undo inappropriate events more than appropriate or neutral ones.

The most common first completion of the "if only" sentence stem was to undo the delay. It was undone more often than each of the other events: the inappropriate controllable event ( $36 \%$ vs. $22 \%$; binomial, $n=44, z=1.5$, $p<.06$ ), the appropriate controllable event ( $36 \%$ vs. $12 \%$; binomial, $n=36, z=3.0, p<.002$ ), the neutral controllable event ( $36 \%$ vs. $11 \%$; binomial, $n=35, z=3.2, p<$ .001 ), and the uncontrollable event ( $36 \%$ vs. $9 \%$; binomial, $n=34, z=3.4, p<.0005$ ).

The results of this experiment are consistent with the idea that the controllability effect may be mediated by whether the events are normal or exceptional with respect to interpersonal norms, because inappropriate controllable events are more mutable than neutral or appropriate controllable events, which are only as mutable as events that are uncontrollable. This finding contrasts somewhat with Girotto et al.'s finding ( 1991 , Experiment 2) that varying whether controllable events were exceptional or normal with respect to intrapersonal norms (i.e., whether the events adhered to or deviated from the person's habits) did not affect their mutability. In the second experiment, we aimed to show that interpersonal and intrapersonal normality are indeed distinct, as suggested by these results and those of Girotto et al.

\section{EXPERIMENT 2}

In our second experiment, our main aim was to show that the effects of interpersonal norms found in the first experiment are distinct from the effects of intrapersonal norms. Gavanski and Wells (1989) found that when an outcome was described as exceptional, participants focused on exceptional antecedent events in their counterfactuals, but that when the outcome was described as normal, they focused instead on normal antecedent events. They gave their participants a scenario about Andrea, a student taking an examination. For all of their participants, the outcome of the scenario was the same: Andrea failed the exam. However, one group was told that Andrea was a poor student, so the outcome was normal with respect to her previous performance. The other group read that Andrea was a good student, and so with respect to her previous performance, the outcome was exceptional. In each version of the scenario, two of the events leading up to the outcome were described as a normal part of Andrea's exam preparation and two were described as exceptional. They called the effect a "norm correspondence effect." Gavanski and Wells suggested that this effect occurs because people tend to assume that there is a correspondence between outcomes and their causes (see, e.g., Einhorn \& Hogarth, 1986; Nisbett \& Ross, 1980). For example, people assume that negative outcomes are caused by negative antecedent events. Gavanski and Wells suggested that people assume that exceptional outcomes are caused by exceptional antecedent events and normal outcomes are caused by normal antecedent events. Therefore, when people want to undo an exceptional outcome, they focus on antecedent events that were exceptional and when they undo a normal outcome they focus on antecedents that were normal.

Gavanski and Wells (1989) have shown that this effect occurs for events and outcomes that are exceptional or normal with respect to intrapersonal norms (the protagonist's habitual examination performance). We suggest that events that are normal or exceptional with respect to interpersonal norms will not show the norm correspondence effect when they are paired with outcomes that differ in their intrapersonal normality, as in previous experiments. Therefore, inappropriate events will be undone more often than appropriate events regardless of whether the outcome of the scenario is normal or exceptional with respect to intrapersonal norms (i.e., habit).

Gavanski and Wells (1989) found a correspondence between the normality of the outcome of a scenario and the events people come to mutate. Likewise, Bouts, Spears, and Van der Pligt (1992) showed that people are more likely to focus on negative antecedent events to undo negative outcomes and on positive antecedent events to undo positive outcomes. In our second experiment, we also tested an alternative hypothesis, that the effects of varying appropriateness on the mutability of controllable events were due instead to a valence correspondence (see also Klauer, Jacobsen, \& Migulla, 1995). The outcome of the scenario that we used in the first experiment and the outcome of the scenario used by Girotto et al. (1991) were negative. It is possible that inappropriate events (such as going into a bar for a beer) are seen as negative and that appropriate events (such as visiting your parents) are seen as positive. Therefore, in our experiment, participants might have been focusing on the inappropriate event instead of the appropriate event because it was negative, as was the outcome of the scenario. In order to rule out this potential explanation of the results of our experiment, we used not only scenarios with negative outcomes, but also scenarios with positive outcomes. If participants were focusing on inappropriate events in the previous experiment because of a valence correspondence effect, we expected that they would instead focus on appropriate events, given a scenario with a positive outcome. However, if this valence correspondence explanation did not hold, we expected that inappropriate events would be mutated more often than appropriate events for both negative (failure) outcomes and positive (success) outcomes.

\section{Method}

Materials pretest. We used a pretest to establish two appropriate and two inappropriate controllable events to include in the scenario. Of these, one of each inhibited success, and the other facilitated success. For the inhibitory events, participants were presented with six events which could have stopped someone from being on time: going for a drink, buying a newspaper, posting a letter, buying cigarettes, picking up a book from the library, stopping for fast food. Their task was to rank the six events in the order in which they be- 
lieved that they were acceptable excuses for being late. For the facilitatory events, they were presented with six events, each of which could be a way of making up lost time: taking a shortcut, going through a stop sign, overtaking a slow driver, ignoring a red light, taking a ring road, driving above the speed limit. Their task was to rank the six events in the order in which they believed them to be acceptable ways to make up time. The participants received the two ranking tasks in random order. The 28 undergraduate students ( 21 women and $7 \mathrm{men}$ ) from the University of Dublin, Trinity College, who took part in this pretest voluntarily were 21 years old on the average.

For each event, a low mean rank indicated that participants found the event to be appropriate (the ranks could range from 1 to 6) and a high mean rank indicated that they found it to be inappropriate. We included in the scenario the inhibitory event of stopping to post a letter, ranked as the most appropriate (mean rank, 1.9), and the inhibitory event of stopping to buy cigarettes, ranked as the (second) least inappropriate (mean rank 4.1), and the difference in rankings for these two events was reliable (Wilcoxon, $z=4.093, p<.0001$ ). ${ }^{2}$ We included the facilitatory event, taking a shortcut, ranked as most appropriate (mean rank, 1.4), and the facilitatory event, driving through a red light, ranked as least appropriate (mean rank, 5.6), and the difference in rankings for the two events was reliable (Wilcoxon, $z=4.713, p<.0001) .^{3}$

Materials and Design. We constructed a scenario based on that in Wells et al. (1987), describing four events that occurred when an individual, Alan, was on his way to a sale in a stereo shop (we chose this scenario because of the ease with which the order of the four antecedents could be varied). Two of the events were inappropriate: One facilitated his successfully getting there on time (running a red light), and one inhibited it (stopping at a tobacconists to buy cigarettes). Two were appropriate: One facilitated his getting there (taking a shortcut), and one inhibited him (stopping to post a letter). The events were chosen to be ones that could be readily carried out in any temporal sequence.

We constructed four versions of the scenario. They differed only in their outcomes in terms of whether or not the protagonist made it to the sale on time (i.e., the valence of the outcome: positive/success or negative/failure), and how exceptional or normal this was for him: exceptional failure, normal failure, exceptional success, or normal success. The normal or exceptional status of success or failure for the protagonist was established by describing his past attempts at getting to the sale on time as either always successes or always failures. An example of one of the scenarios (the exceptional failure scenario) is as follows:

Alan likes music a lot. There is one stereo shop in particular that he frequents. This shop has a good sale on a limited amount of stock once a year. It is very popular and the best deals usually go within the first half hour. Alan tries to make it to the sale on time every year. So far he has always succeeded.

It is the morning of the sale and there are a number of things that Alan wants to do. He gets into his car and leaves home in good time to make it to the sale. Alan starts his morning by taking a shortcut through some side streets. He then goes to the post office to post a letter. Next, Alan stops at a tobacconist's to buy some cigarettes. Coming up to the stereo shop, Alan drives through a red light. Alan arrives at the shop half an hour after the sale starts only to find that the last stereo had just been sold.

Alan is really annoyed, he thinks "Things would have been different if ..."

The participants were asked to provide four likely completions of Alan's thoughts. The experiment had four independent variables: two between subjects, and two within subjects. One between-subjects variable was the valence of the outcome (i.e., success or failure), and the other was the exceptionality of the outcome (i.e., exceptional or normal). One within-subjects variable was the appropriateness or inappropriateness of the target antecedent event, and the other was the facilitatory or inhibitory nature of the target event. The dependent variable was the frequency with which participants mentioned each of the four target events in their first sentence completion.

Participants and Procedure. The participants were 149 students from the University of Dublin, Trinity College (117 women and $32 \mathrm{men}$ ) who took part in the experiment voluntarily. Their average age was 20 . They were randomly assigned to one of the four outcome conditions (exceptional failure, $n=40$; normal failure, $n=$ 37; exceptional success, $n=36$; normal success $n=36$ ). The participants were presented with a page with instructions and space for recording age and gender at the top, followed by one of the versions of the scenario with space for recording four answers. This was followed by the question about whether each answer given had described how things could be better or worse. They were instructed to write down answers as they occurred to them and not to change their answers once they had written them.

\section{Results and Discussion}

First counterfactual alternatives. The participants mutated inappropriate inhibitors for both exceptional and normal failure outcomes, and they mutated inappropriate facilitators for both exceptional and normal success outcomes. They mutated the inappropriate inhibitor for the two failure outcome scenarios more than any other event (see Table 2). For the exceptional failure scenario, the participants changed the inappropriate inhibitor most often overall (e.g., they suggested he would have gotten there on time if he had not stopped at the tobacconist's). They changed it more often than the appropriate inhibitor, posting a letter ( $38 \%$ vs. $13 \%$; binomial, $n=20, z=2.24$, $p<.02$ ); the inappropriate facilitator, running a red light ( $38 \%$ vs. $0 \%$; binomial, $n=15, z=3.87, p<.0001$ ); and the appropriate facilitator, taking a shortcut $(38 \%$ vs. $0 \%$; binomial, $n=15, z=3.87, p<.0001)$. Neither of the facilitators, whether inappropriate (the red light) or appropriate (the shortcut), was ever changed ( $0 \%$ each). The appropriate inhibitor (posting a letter) was changed more often than either of the facilitators $(13 \%$ vs. $0 \%$ in each case; binomial, $n=5, z=2.24, p<.02$ ).

Exactly the same pattern occurred for the normal failure scenario: participants' mutations focused on the inappropriate inhibitor (the cigarettes) most often overall. They changed the inappropriate inhibitor (the cigarettes) more often than the appropriate inhibitor, the letter $(43 \%$ vs. $16 \%$; binomial, $n=22, z=2.13, p<.02)$; the inappropriate facilitator, the red light $(43 \%$ vs. $3 \%$; binomial, $n=17, z=3.64, p<.0002)$; and the appropriate facilitator, the shortcut ( $43 \%$ vs. $0 \%$; binomial, $n=16, z=4$, $p<.00003$ ). Once again, neither of the facilitators, whether inappropriate (the red light) or appropriate (the shortcut) was changed often ( $3 \%$ and $0 \%$, respectively). The appropriate inhibitor, the letter, was changed more often than the inappropriate facilitator, the red light ( $16 \%$ vs. 3\%; binomial, $n=7, z=1.89, p<.03)$, and the appropriate facilitator, the shortcut ( $16 \%$ vs. $0 \%$; binomial, $n=6, z=2.45, p<.008$ ).

The participants mutated the inappropriate facilitator for the two success outcome conditions more often than 
Table 2

The Percentages of Mutations of the Target Events for the Four Different Outcome Scenarios in Experiment 2

\begin{tabular}{lcccccc}
\hline & \multicolumn{2}{c}{ Failure } & & \multicolumn{2}{c}{ Success } & \\
\cline { 2 - 3 } \multicolumn{1}{c}{ Exent } & $\begin{array}{c}\text { Exception Norm } \\
(n=40)\end{array}$ & & $\begin{array}{c}\text { Exception Norm } \\
(n=37)\end{array}$ & & Total \\
$(n=36)$ & $(n=36)$ & $(n=149)$ \\
\hline Inhibitory & & & & & \\
$\quad$ Inappropriate (cigarettes) & 38 & 43 & & 14 & 6 & 26 \\
$\quad$ Appropriate (letter) & 13 & 16 & & 3 & 3 & 9 \\
Facilitatory & & & & & \\
$\quad$ Inappropriate (red light) & 0 & 3 & & 47 & 47 & 23 \\
$\quad$ Appropriate (shortcut) & 0 & 0 & & 8 & 8 & 4 \\
Total & 51 & 62 & & 72 & 64 & \\
\hline
\end{tabular}

any other event. For the exceptional success scenario, the participants' mutations focused mainly on the inappropriate facilitator (e.g., they suggested that he would not have gotten there on time if he had not gone through the red light). They changed the inappropriate facilitator, the red light, more often than the appropriate facilitator, the shortcut $(47 \%$ vs. $8 \%$; binomial, $n=20, z=3.13, p<$ $.001)$; the inappropriate inhibitor, the cigarettes $(47 \%$ vs. $14 \%$; binomial, $n=22, z=2.56, p<.006)$; and the appropriate inhibitor, the letter ( $47 \%$ vs. $3 \%$; binomial, $n=$ $18, z=3.77, p<.0001$ ). They changed the appropriate facilitator, the shortcut, as often as the appropriate inhibitor, the letter ( $8 \%$ vs. $3 \%$; binomial, $n=4, z=1, p<.16)$, and the inappropriate inhibitor, the cigarettes $(8 \%$ vs. $14 \%$; binomial, $n=8, z=0.71, p<.24)$. They showed a small tendency to undo the inappropriate inhibitor, the cigarettes, more often than the appropriate inhibitor, the letter, although the difference was statistically marginal ( $14 \%$ vs. $3 \%$; binomial, $n=6, z=1.63, p<.06$ ).

Exactly the same pattern occurred for the normal success scenario: The participants' mutations again focused on the inappropriate facilitator (the red light). They changed the inappropriate facilitator, the red light, more often than the appropriate facilitator, the shortcut $(47 \%$ vs. $8 \%$; binomial, $n=22, z=3.13, p<.001)$; the inappropriate inhibitor, the cigarettes $(47 \%$ vs. $6 \%$; binomial, $n=19, z=3.44, p<.0003)$; and the appropriate inhibitor, the letter $(47 \%$ vs. $3 \%$; binomial, $n=18, z=3.77, p<$ $.0001)$. They changed the appropriate facilitator, the shortcut, as often as the inappropriate inhibitor, the cigarettes ( $8 \%$ vs. $6 \%$; binomial, $n=5, z=0.45, p<.33$ ), and the appropriate inhibitor, the letter $(8 \%$ vs. $3 \%$; binomial, $n=4, z=1, p<.16$ ). They changed the inappropriate inhibitor, the cigarettes, as often as the appropriate inhibitor, the letter ( $6 \%$ vs. $3 \%$; binomial, $n=3, z=0.58$, $p<.29$ ).

The results show that for failure outcomes, the participants focused on the inappropriate inhibitor in their mutations, suggesting that things would have been different (i.e., he would have gotten there on time) if he had not stopped at the tobacconist's to buy cigarettes. For success outcomes, they focused instead on the inappropriate facilitator, suggesting that things would have been different (i.e., he would not have gotten there on time) if he had not gone through the red light. The participants focused on inappropriate controllable antecedents more than the appropriate controllable ones, for all outcomes ( $49 \%$ vs. $13 \%$; binomial, $n=84, z=3.27, p<.0006$ ).

Alternative explanations. Overall, then, the results of the experiment show that the mutability effect that we found in the first experiment (that inappropriate controllable events are more mutable than appropriate controllable events) is distinct from the exceptionality effect studied by other researchers (e.g., Gavanski \& Wells, 1989; Girotto et al., 1991; Kahneman \& Tversky, 1982). If they were one and the same thing, participants would focus on inappropriate events when the outcome of the scenario was described as exceptional and on appropriate events when the outcome of the scenario was described as normal; that is, they would follow a norm correspondence pattern (Gavanski \& Wells, 1989). However, the experiment shows that participants change inappropriate events more often than appropriate events for exceptional outcomes ( $49 \%$ vs. $12 \%$; binomial, $n=46, z=4.6, p<.00003$ ), and they also change inappropriate events more often than appropriate events for normal outcomes ( $49 \%$ vs. $14 \%$; binomial, $n=46, z=4.33, p<.00003$ ).

The results also rule out the idea that inappropriate events are more mutable because of a valence correspondence effect: this explanation predicts that participants should change inappropriate events when the outcome is negative (failure) and appropriate events when the outcome is positive (success). The experiment shows that participants undo inappropriate events more often than appropriate events for negative (failure) outcomes ( $42 \%$ vs. $14 \%$; binomial, $n=43, z=3.71, p<.0001)$, and that they also undo inappropriate events more often than appropriate events even for positive (success) outcomes (57\% vs. $11 \%$; binomial, $n=49, z=5.15, p<.00003$ ).

Finally, the results rule out the idea that inappropriate events are more mutable because of an interaction between normality and valence: This explanation predicts that people should undo inappropriate events for outcomes that are both negative and exceptional, and they should undo appropriate events for outcomes that are both positive and normal. The experiment shows that people undo inappropriate events more than appropriate events for negative exceptional outcomes ( $38 \%$ vs. $13 \%$; bino- 
mial, $n=20, z=2.24, p<.02$ ), but that they also change inappropriate events more than appropriate events even for positive normal outcomes ( $53 \%$ vs. $11 \%$; binomial, $n=23, z=3.13, p<.001$ ).

\section{GENERAL DISCUSSION}

An inappropriate controllable event is more mutable than an uncontrollable event, as Girotto et al. (1991) have shown. However, the results of the two experiments reported here show that there are differences in the mutability of controllable events, depending on their appropriateness. The first experiment shows that people undo inappropriate controllable events more often than appropriate controllable events. They undo appropriate and neutral controllable events no more often than an uncontrollable event. The second experiment shows that inappropriate events are mutated not only for scenarios that end with a negative and exceptional outcome, but also for other scenarios with positive outcomes and with normal outcomes.

The two experiments have shown that not only can the interpersonal norm/exception status of events affect their mutability, but also this effect differs from the exceptionality effect that has been previously reported in the literature (e.g., Gavanski \& Wells, 1989; Kahneman \& Tversky, 1982). The results suggest that research needs to address the two possible kinds of normality: intrapersonal normality - whether or not people adhere to their habitual modes of behavior - and interpersonal normalitywhether or not people abide by prevailing social norms. Both intrapersonal and interpersonal normality can affect the counterfactual mutations that people make. Previous research has focused only on intrapersonal normality, and we suggest that interpersonal normality is equally important. We have shown that the appropriateness of events can affect their mutability. However, we consider that this is not the only way in which events can deviate from prevailing social norms. Events may be seen as more or less necessary, selfish, legal, and so on. There are many other possibilities when it comes to social constraints on counterfactual thinking about what might have been, and they have yet to be fully explored.

One possible explanation for the controllability effect and the role of the appropriateness of events may be the nature of the mental representations that people construct when they generate a counterfactual alternative. One suggestion has been that the counterfactual alternatives that people most readily construct depend on what is represented explicitly in the mental models that they construct for the factual situation (e.g., Byrne, 1997; Byrne et al., 2000; McCloy, 2000). When people think about an inappropriate controllable event they may construct a model of the inappropriate event, and they may also keep in mind a model of the more appropriate event (see also Kahneman \& Miller, 1986; Legrenzi, Girotto, \& Johnson-
Laird, 1993). When they generate a counterfactual alternative to the inappropriate event, the model of the more appropriate event is readily available as a candidate. In contrast, when they think about an appropriate controllable event, they may keep in mind just a single model corresponding to it. Because no counterfactual alternative model is readily available, the appropriate controllable event is then less likely to be mutated.

The "counterfactual fallacy" is the tendency to think that what need not have been ought not have been (Miller, Turnbull, \& McFarland, 1990); that is, people treat epistemic necessity as if it implied deontic necessity. The results of our experiments indicate that people may also sometimes exhibit the converse of the counterfactual fallacy: They think that what ought not have been need not have been.

\section{REFERENCES}

Bouts, P., Spears, R., \& Van der Pligt, J. (1992). Counterfactual processing and the correspondence between events and outcomes: Normality vs. value. European Journal of Social Psychology, 22, 387-396.

BYRNE, R. M. J. (1997). Cognitive processes in counterfactual thinking about what might have been. In D. L. Medin (Ed.), The psychology of learning and motivation: Advances in research and theory (Vol. 37, pp. 105-154). San Diego: Academic Press.

Byrne, R. M. J., Segura, S., Culhane, R., Tasso, A., \& Berrocal, P. (2000). The temporality effect in counterfactual thinking about what might have been. Memory \& Cognition, 28, 264-281.

ByRne, R. M. J., \& TASso, A. (1999). Deductive reasoning with factual, possible, and counterfactual conditionals. Memory \& Cognition, 27, 726-740.

EINHORN, H. J., \& HogarTh, R. M. (1986). Judging probable cause. Psychological Bulletin, 99, 3-19.

GavanSKI, I., \& WeLLS, G. L. (1989). Counterfactual processing of normal and exceptional events. Journal of Experimental Social Psychology, 25, 314-325.

Girotto, V., Legrenzi, P., \& Rizzo, A. (1991). Event controllability in counterfactual thinking. Acta Psychologica, 78, 111-133.

Kahneman, D., \& Miller, D. T. (1986). Norm theory: Comparing reality to its alternatives. Psychological Review, 93, 136-153.

KAHNEMAN, D., \& TVERSKy, A. (1982). The simulation heuristic. In D. Kahneman, P. Slovic, \& A. Tversky (Eds.), Judgment under uncertainty: Heuristics and biases (pp. 201-211). Cambridge: Cambridge University Press.

Klauer, K. C.. Jacobsen, T., \& Migulla, G. (1995). Counterfactual processing: A test of a hierarchical correspondence model. European Journal of Social Psychology, 25, 577-595.

Legrenzi, P., Girotto, V., \& Johnson-Laird, P. N. (1993). Focusing in reasoning and decision making. In P. N. Johnson-Laird \& E. Shafir (Eds.), Reasoning and decision making (pp. 37-66). Oxford: Blackwell.

LEWIS, D. (1973).Counterfactuals. Oxford: Blackwell.

MANDEL, D. R., \& LEHMAN, D. R. (1996). Counterfactual thinking and ascriptions of cause and preventability. Journal of Personality \& $S o$ cial Psychology, 71, 450-463.

MCCLoY, R. (2000). Thinking about what might have been: Cognitive processes in counterfactual and semifactual thinking about controllable events. Unpublished doctoral thesis, University of Dublin, Trinity College.

Miller, D. T., \& Gunasegaram, S. (1990). Temporal order and the perceived mutability of events: Implications for blame assignment. Journal of Personality \& Social Psychology, 59, $1111-1118$.

Miller, D. T., Turnbull, W., \& McFarland, C. (1990). Counterfac- 
tual thinking and social perception: Thinking about what might have been. In P. Zanna (Ed.), Advances in experimental social psychology (Vol. 22, pp. 305-331). San Diego: Academic Press.

N'gbala, A., \& Branscombe, N. R. (1995) Mental simulation and causal attribution: When simulating an event does not affect fault assignment. Journal of Experimental Social Psychology, 31, 139-162.

NisBeTt, R. E., \& Ross, L. (1980). Human inference: Strategies and shortcomings of social judgment. Englewood Cliffs, NJ: PrenticeHall.

ROESE, N. J. (1997). Counterfactual thinking. Psychological Bulletin, 121, 133-148.

RoEse, N. J., \& OLSON, J. M. (EDS.) (1995). What might have been: The social psychology of counterfactual thinking. Hillsdale, NJ: Erlbaum.

Seelau, E. P., Seelau, S. M., Wells, G. L., \& Windschitl, P. D. (1995). Counterfactual constraints. In N. J. Roese \& J. M. Olson (Eds.), What might have been: The social psychology of counterfactual thinking (pp. 57-79). Hillsdale, NJ: Erlbaum.

STALNAKER, R. C. (1968). A theory of conditionals. In N. Rescher (Ed.), Studies in logical theory (pp. 98-112). Oxford: Blackwell.

Wells, G. L., Taylor, B. R., \& TurTlE, J. W. (1987). The undoing of scenarios. Journal of Personality \& Social Psychology, 53, $421-430$.

\section{NOTES}

1. Drinking alone, the controllable event used by Girotto et al. (1991), was ranked as joint fourth most inappropriate event.

2 . We did not choose the most inappropriate inhibitory event, stopping for a drink (mean rank, 5.9), because drinking and driving may be a particularly salient inappropriate event, being the subject of various health and safety promotion campaigns.

3. The order in which the inhibitory events were ranked was as follows: posting a letter (mean rank, 1.9), picking up a book from the library (2.4), stopping for fast food (3.3), buying a newspaper (3.6), buying cigarettes (4.1), and going for a drink (5.9). The order in which the facilitatory events were ranked was as follows: taking a shortcut (mean rank, 1.4), taking a ring road (2.0), overtaking a slow driver (2.9), driving above the speed limit (4.3), going through a stop sign (4.8), and ignoring a red light (5.6).

(Manuscript received April 7, 1999; accepted for publication October 4, 1999.) 